\title{
The effects of washing without water versus the traditional bed bath with water and soap on comfort and physical demands: protocol of a cross- over randomized trial
}

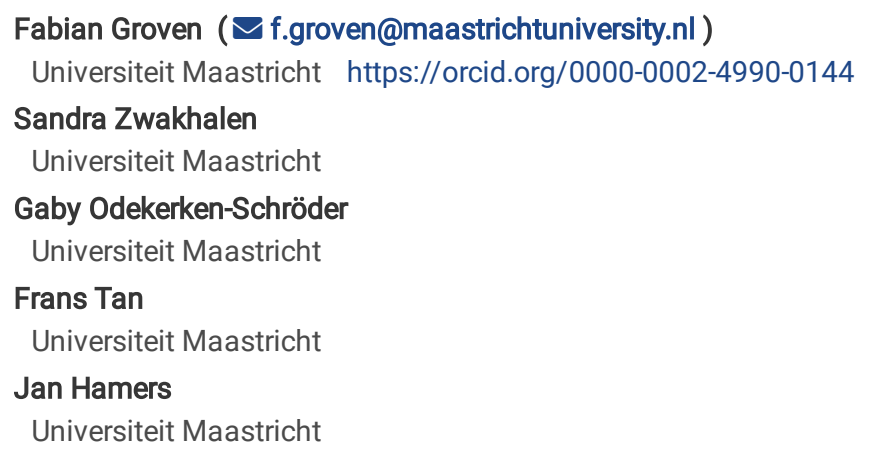




\section{Abstract}

Background The traditional bed bath with water and soap often leads to emotional and physical discomfort among bedridden patients. In addition, the traditional bed bath is physically demanding for nurses. The washing without water concept is increasingly used in healthcare to bathe bedridden patients and is likely to affect emotional and physical comfort dimensions. This protocol describes a study that will assess how washing without water performs compared to the traditional bed bath regarding emotional and physical comfort experienced by the bathed bedridden person and regarding physical demands for the provider of the bed bath.Methods This study uses a randomized cross-over design in which nursing students will simulate the bed baths of patients in a laboratory setting. Students will be randomly allocated to a patient or a nurse group and consequently will respectively receive or provide two bed baths: the traditional bed bath and a washing without water bed bath. The order of the bed baths will be randomly determined. Students will receive instructions about the steps of the bathing methods and case descriptions about their role as a patient or nurse. Questionnaires will be used before and after each bed bath to collect data for the primary outcomes of experienced emotional and physical comfort (among students receiving the bed bath) and physical demands (among students providing the bed bath); and, for secondary outcomes related to bed bathing preferences, grades given to the bathing methods and perceived importance of the bed bath. Differences between the two bathing methods will be analyzed, taking the order of the bed baths into account.Discussion This study will add to the scientific knowledge of the washing without water bed bath, for which evidence on comfort is currently lacking. If differences in comfort between washing without water and the traditional bed bath are detected among nursing students, it is likely these differences will also be present among actual patients and nurses, as generally, patients are more vulnerable and nurses spend more time bathing patients compared to students.

\section{Background}

Hygiene is described to be one of the essential elements of care that nurses need to provide to persons who cannot bathe independently anymore. Whereas bathing is a self-activity that ensures health and wellbeing for independent people, bathing often leads to physical and emotional discomfort among persons who cannot bathe independently anymore and, hence, need assistance in bathing [1, 2]. Emotionally, assisted bathing can contribute to comfort but often is experienced by patients as an intrusive encounter [3].

Bedridden patients require assistance in bathing most often. The bed bath has been defined as "an episode of nursing care where ... patient comfort is provided" (p. 201) [4]. However, the limited evidence on bed bathing shows that bedridden patients often perceive the bed bath to have a detrimental effect on their level of physical and emotional comfort. The traditional bed bath with water and soap has been perceived as embarrassing, dependent, uncomfortable, and difficult among others [5]. Moreover, the bed bath with water and soap is physically demanding for both patients and nurses. Nevertheless, most bedridden patients are still bathed with water and soap.

The washing without water concept is increasingly used in healthcare practice as an alternative for the traditional bed bath with water and soap. The concept can be described as disposable, prepacked products for the full body wash consisting of a nonwoven carrier (washcloth) and a no-rinse cleansing fluid that allows nursing staff to bathe someone without the use of water [6]. Results of a systematic review showed washing without water is a worthy alternative for the traditional bed bath with water and soap. However, the number of studies on washing without water is limited and evidence for patient-related variables, such as physical or emotional comfort is lacking [7].

Nevertheless, it is very likely that washing without water affects the comfort of patients [8]. Washing without water can contribute or jeopardize experienced patient comfort directly, both physically and emotionally. Physical comfort pertains to bodily sensations [9], which in case of a bed bath will probably be influenced by the bathing method applied. Furthermore, washing without water is expected to impact the emotional comfort of patients, which is defined as "pleasant positive feelings, a state of relaxation that affected the physical status of the body" (p.809) [10]. In one study, the majority of patients indicated they would permanently replace water and soap with washing without water [6]. It is possible that the preferences of patients are explained by higher levels of perceived comfort, as nurses reported a preference for washing without water when asked about patient comfort in another study [11].

Washing without water can also make a substantial contribution to experiences of nurses, as bathing assistance will remain a highly prevalent nursing activity [1]. Evidence shows that the duration of the bed bath with washing without water is shorter compared to the traditional bed bath with water and soap. The reduced time consumption as well as the convenience of the concept have been reported to be reasons for nurses to prefer washing without water to the traditional bed bath [7]. In addition, washing without water is argued to be less physically burdensome for nurses. However, more evidence on physical demands for nurses is required, as this is only studied in unpublished studies [12, 13].

The objective of this study is to assess how washing without water performs compared to the traditional bed bath, with respect to experienced emotional and physical comfort dimensions. Based on previous studies, the research hypothesis is that washing without water is experienced to be 1) equally or more emotionally and physically comfortable by the bathed bedridden person and 2) equally or less physically demanding by the provider of the bed bath. Our study will not only add to the evidence on washing without water but also contribute to the essential care concept (often referred to as fundamental or basic care). Bed bathing belongs to "personal cleansing and dressing," which is one of the physical needs addressed by essential care [14]. Feo and Kitson [2] argue that nurses generally undervalue essential care; therefore, bathing is often delivered below par. Our findings regarding washing without water on comfort can contribute to better bed bathing experiences and, in turn, to the revaluation of bathing as part of essential care. 


\section{Methods}

\section{Study design and participants}

This study uses a randomized cross-over design in which the bed baths of patients are simulated in a laboratory setting. Participants (nursing students) will be randomly allocated to a patient or a nurse group and consequently will respectively receive or provide both the intervention, being a bed bath with a washing without water product, and the control bed bath with water and soap. The order of the bed baths will be randomly determined. From now on, students assigned to the patient or the nurse group are called "patient students" and "nurse students," respectively. All patient students and nurse students together are referred to as "participants."

Participants will be recruited out of nursing students of several Dutch intermediate and higher vocational educational institutions in a timespan of 2 to 3 years. The study will be executed at the educational institutions in rooms equipped as nursing rooms containing patient beds, bed trolleys and bed curtains. Data will be collected immediately before and after each bed bath. The data will mainly be quantitative, but some qualitative data will also be collected, which will be used to interpret the findings of this study. This study and protocol are based on the "Standard Protocol Items: Recommendations for Interventional Trials" (SPIRIT) statement [15]. Finally, ethical approval has been obtained by a Dutch medical committee (17-N172), and the trial is registered at www.trialregister.nl (6972).

\section{Inclusion and exclusion criteria}

The study sample will consist of nursing students representing bedridden patients and nurses. All first and second year nursing students are eligible. To be included in the study, students should be: 1) willing to bathe fellow students and 2) willing to receive a bed bath in swimming or underwear (allowed to respect the privacy of students) by fellow students. Only first and second year nursing students are included, because these students are likely to lack much practical experience with bed bathing and, therefore, have to attend practice lessons in bathing. No specific exclusion criteria have been determined.

\section{Recruitment and randomization}

Participants will be recruited in close collaboration with the coordinators of first and second year nursing studies at the particular educational institutions. To increase the participation rate, this study will be combined with practical bathing lessons, which are part of the curricula of first and second year nursing studies in which all students will also be instructed about the different bed bathing methods. Furthermore, in lectures all first and second year nursing students will be informed about the trial after which students have the opportunity to ask questions. Students will then receive an information letter accompanied by an informed consent form and an eligibility questionnaire to assess whether students meet the inclusion criteria. Students can hand in the signed form of consent and the completed eligibility questionnaire immediately or within one week after the lecture. Finally, a vlog about the research will be shared with all first and second year nursing students a few days after the lectures to increase participation. Acceptance or refusal to participate will not affect students' study results in any way.

The first author will assess if students who gave informed consent meet the inclusion criteria. Subsequently, unique identifier numbers will be assigned to all eligible students to ensure confidentiality. The identifier numbers will be used to assign participants to the patient or the nurse group, to determine the order of bed baths, and to assign a room and timeslot to participants by using a computer-generated randomization table. Half of the participants receive or provide the washing without water bed bath (intervention) first, whereas the other half starts with the bed bath with water and soap (control). A 1:1 ratio will be used to ensure an equal amount of patient students and nurse students within each group, as every patient student needs a nurse student to provide the bed baths. To keep other factors than the bed bath method as constant as possible, each patient student will receive both bed baths from the same nurse student, at the same time of the day, and in the same bed and room (at each educational institution, between 14 and 20 beds will be available spread across four to six rooms equipped as a nursing room). A wash-out period of one week will be included between the two bed baths.

Before the start of the trial, participants will electronically receive information about the time and place of the bed baths. Furthermore, participants will receive their unique identification number, which they need to write down in every questionnaire used for data collection in this study. Finally, participants will be informed about the role assigned to them (patient or nurse) and the bed bath order (the washing without water bed bath or the traditional bed bath first) before the start of the trial. Figure 1 shows the participant timeline based on the "Consolidated Standards of Reporting Trials" (CONSORT) flow diagram [16], including the bed bath order for the different groups of patient and nurse students.

Legend to Figure 1: " $w-w$ " is a washing without water bed bath and " $w+w$ " stands for the traditional bed bath with water and soap. Groups $1 A$ and $2 A$ (patient students) and 1B and 2B (nurse students) each differ with respect to the order in which the two bed bathing methods take place. In groups $1 \mathrm{~A}$ and $1 B$, students start with the washing without water bed bath, whereas groups $2 \mathrm{~A}$ and $2 \mathrm{~B}$ start with the bed bath with water and soap. 
The intervention in this study is a washing without water bed bath that uses a disposable, prepacked product for the full body wash consisting of eight nonwoven gloves with a no-rinse, fragranced cleansing fluid [6]. The traditional bed bath approach with washbasins, water, soap, towels, and washing mitts will be used in the control group, as this is still the most adopted bed bathing method in healthcare.

To ensure uniform execution of the bed baths, participants will receive verbal and written step-by-step instructions for both bed bathing methods (instructions are available upon request). Apart from the methods used, the instructions of the washing without water bed bath and the traditional bed bath are highly similar. The instructions are aligned with the educational institutions and developed for this study based on bathing protocols obtained from the Internet (for the control bed bath) [17] and from a washing without water supplier (for the intervention bed bath). Research assistants will observe the bed baths for compliance with the instructions.

In addition to the bathing instructions, participants will obtain instructions related to the role assigned to them. Patient students will receive a case description, which describes the case of a patient who broke his or her right leg. Hence, instructions are provided about which consequences this injury has for the steps during the bed bath (e.g., they can wash their face themselves but need help in sitting up). Nurse students are instructed to confine communication during the bed bath and will be provided with a case description that explains to them that the patient they are about to bathe is the second of many patients that need to be bathed in a limited period. All patient students and all nurse students will receive the same case descriptions without any variation. Although case descriptions cannot mimic reality completely, these instructions at least to some extent simulate the situations real patients and nurses encounter [18].

\section{Data collection and measures}

Data will be collected by paper questionnaires that all participants need to fill out right before and immediately after each bed bath. Hence, for each bed bath, we have two baseline (B) questionnaires (one for patient students and one for nurse students) that need to be filled out before the bed bath, and two outcome (0) questionnaires that need to be filled out after the bed bath; again, one for each group of participants. Table 1 shows which outcomes are included in the questionnaires before each bed bath (B1 and B2 in Table 1 for the questionnaire before the first and the second bed bath, respectively) and in the questionnaires after each bed bath (01 and 02) for each of the participant groups. The group of patient students and nurse students will fill out the questionnaires in separate rooms. Participants have 30 minutes for one bed bath, excluding the completion of the baseline and outcome questionnaires, which will take approximately 15 minutes.

\section{Demographic data}

Demographic data of all participants will be collected at baseline (B1) to describe the study sample, including age, weight, length, gender, educational level, and experience with bed baths obtained before the trial.

\section{Primary outcome data}

The primary outcomes related to patient students are experienced emotional and physical comfort, which will be measured using validated scales. To measure emotional comfort, the 12-item Patient Evaluation of Emotional Comfort Experienced (PEECE) scale will be used [19]. Patient students need to indicate to what extent they experienced certain feelings during the bed bath (relaxed, valued, safe, calm, cared for, at ease, like smiling, energized, content, in control, informed, thankful) on a five-point scale. The cumulative score will be used in the analysis, ranging from 12 to 60 ; a high score meaning a high level of experienced emotional comfort. High Cronbach a coefficients (between 0.74 and 0.88 ) have been reported for the PEECE scale in previous studies [19]. Physical comfort will be measured by a single item question asking the patient student about the perceived comfort of the bed bath on a ten-point Likert scale ranging from "very uncomfortable" to "very comfortable".

The physical demands scale [20] will be used to measure physical demands imposed by the bed bath on nurse students. The scale consists of seven items related to physically demanding activities (e.g., carrying or moving weights heavier than 10 kilograms) or postures (e.g., standing for a long time). Nurse students have to indicate to what extent these activities and postures were present during the bed bath on a five-point Likert scale. The cumulative score will be used for the data analysis, ranging from seven to 35; a high score meaning high physical demands. Previous studies that used the physical demands scale reported high Cronbach a coefficients of 0.87 [21] and 0.91 [20], respectively.

\section{Secondary outcome data}

In addition to the primary outcomes, several measures are related to the secondary outcomes of this trial: 
- To assess emotional comfort for patient students perceived by nurse students, the PEECE scale is also filled out by nurse students.

- Physical comfort for patient students as perceived by nurse students is measured by a single item question similar to the question about physical comfort asked to patient students directly.

- The bed bathing method preference among patient students will be measured in several ways. Patient students will be asked directly which bathing method they prefer after they received both the intervention and control bed bath. Furthermore, after each bed bath, patient students are asked to give an overall grade to the bathing method they just received, ranging from one (very bad) to ten (very good). Finally, after both bed baths have been received, patient students are asked how they would like to be bathed in the future.

- In addition, nurse students will be asked directly for their bathing method preference and for an overall grade for both bathing methods. Finally, after both bed baths have been provided, nurse students are asked which type of bed bath they would like to provide in the future.

- The perceived importance of the bed bath according to all participants (patient and nurse students) will be measured by using a numeric rating scale from one (being not important at all) to 10 (being extremely important).

- The duration of the bed bath will be calculated using the start and end time of each bed bath as recorded by the research assistants observing the bed baths.

Process evaluation

In addition to the primary and secondary outcomes, the process of some of the bed baths provided during the trial will be evaluated by research assistants with a background in nursing who will mainly focus on fidelity (if the bed baths were conducted as planned). Furthermore, short interviews with a sample of participants will be held to gather experiences with both bathing methods and to assess the satisfaction of participants with the trial. The qualitative data will be used in the interpretation of the outcome findings.

\section{Participant retention}

The trial will be combined with practice lessons in bathing, which are part of the regular curriculum of first and second year nursing students. Furthermore, participants will fill out the questionnaires right before and immediately after each bed bath. Hence, measures to promote participant retention and to complete follow-up are not necessary. However, it is likely participants will drop out due to the wash-out period and because they may choose to withdraw from the trial. To increase the chance of complete follow-up, participants will frequently be informed and reminded of the study through lectures and information via email or on the electronic learning environment with support of the educational institutions.

\section{Reliability}

The laboratory setting offers opportunities to increase fidelity among participants. Participants will receive elaborate instructions including a step-bystep description of the intervention and the control bed bath. In addition, participants will only have the bathing materials at their disposal that are needed for the particular bed bath they are expected to receive or provide. Hence, intention to treat analyses will not be needed for this study.

Reliability will also be taken into account in the data management. Referential data rules, valid values, and range checks will be performed to check the data. Furthermore, the data will be checked for outliers, and a second researcher will inspect a subset of the data to detect possible errors.

\section{Power and sample size}

A power calculation for paired measurements [22] shows that in total at least 104 participants should be recruited; half of them being patient students, half of them being nurse students. The sample size is based on the primary outcome of emotional comfort, with an effect size of 0.5 , a type I error of 0.05 and $95 \%$ power. First and second year nursing students (at least 700 in total) of the participating educational institutions will be contacted to participate in the trial, which is expected to be sufficient given the assumed attrition rate of $15 \%$.

\section{Data analysis}

In case we don't have missing values, a one sample t-test will be executed to test for the significance of the change in scores between the two bathing methods with respect to experienced emotional and physical comfort, physical demands, overall grades, and bed bath duration. Additionally, a one sample t-test will be performed to test for differences in perceived importance of the bed bath before and after the trial. Instead, a two-level regression analysis [23] will be applied in the case of missing values, with repeated measurements on the first level and participants on the second level. Under the assumption of missing completely at random, a two-level regression analysis offers an unbiased and more efficient method compared to the one sample t-test on change in scores [24]. Preferences for bed bath methods will be tested by using a simple binomial test.

Page 5/10 
Because of the cross-over design, additional analyses will be executed to test whether the dependent outcomes (experienced emotional and physical comfort, physical demands, overall grades, bed bath duration, and importance of the bed bath) differ depending on the order of the bed baths. Hence, the order of the bed bath will be included as a dummy variable in separate regression analyses together with time and an interaction variable between time and order of the bed bath. Finally, the influence of the bed bath order on preferences for bathing methods will be tested using the direct likelihood method.

Possibly, some variables will be included as covariate in the regressions if enough variation on the particular variables is found within the study sample. Potential covariates for which data will be collected during the trial are:

- Educational level of the participating nursing students (possible covariate of all outcomes)

- Emotional comfort experienced by the patient student before the bed bath (possible covariate of experienced emotional and physical comfort of the bed bath);

- Performance assessment by the patient student of how the nurse student executed the bed bath (possible covariate of experienced emotional and physical comfort of the bed bath and the grade given to the bed bath);

- Self-assessment by the nurse student (possible covariate of emotional and physical comfort of the bed bath as perceived by the nurse student);

- Physical complaints before the bed baths among all participants (possible covariates of experienced physical comfort of the bed bath and physical demands of the bed bath);

- Knowing or not knowing the patient or the nurse student (possible covariate of experienced emotional comfort).

Finally, additional analyses will be conducted to detect possible differences in baseline measurements between the different groups (nurse and patient students, participants starting with the intervention or the control bed bath, participants who completed the trial and dropouts, and nursing students from different educational institutions). All randomized participants will be included in the main analyses, regardless of protocol adherence. SPSS 25 will be used for the data analysis. Confidence intervals of 0.05 will be applied, and the results will be presented with a $5 \%$ threshold.

\section{Data monitoring and management}

The data will be stored on a central and secured directory, which is only accessible by the research team for at least 10 years after last publication. Furthermore, as the data collection will only take two days for each group of students (excluding the wash-out period) and the risks for participants during the trial are basically non-existent, there is no need for periodic inspection of accumulating data to make adjustments accordingly.

\section{Discussion}

The objective of this study is to measure the effects of the washing without water bed bath on experienced emotional and physical comfort of persons being bathed and on physical demands of the washing without water bed bath among persons providing the bed bath. Despite that the bed bath in general has been reported to often have a detrimental impact on comfort, the experiences of patients and nurses with washing without water regarding comfort have not been thoroughly assessed. Therefore, this study's results offer a valuable contribution to the scientific knowledge on the washing without water bed bath. We think this knowledge will increase the awareness among healthcare providers of the impact of the bed bath on patients and consequently will increase the perceived importance of this essential nursing care activity.

This study's cross-over design, reduces the risk of bias, allows a smaller sample size [25], and gives participants the possibility to experience both types of bed baths, either as a patient or as a nurse. Moreover, the cross-over design is most suitable to measure preferences for bathing methods, which is included as a secondary outcome, because participants will experience both types of bed baths [26]. We want to comment on two often underreported methodological issues related to cross-over trials, which are allocation concealment and carryover effects [26]. In this study, the allocation of participants to the different groups will not be concealed, as blinding of participants to the different bed bathing methods is impossible. Furthermore, carryover effects are unlikely, because the effects of the bed baths are short-lived and because of the one week wash-out period.

A laboratory setting was chosen to be able to measure causation between the bed bath and comfort by isolating the bed bath from other nursing activities that might influence comfort as well. It would be very difficult to assess the effects of the bed bathing method on comfort in a natural setting, in which the bed bath method is only one of many uncontrollable nursing elements [27]. Our laboratory experimental design enables a high internal validity because of the control of possible confounding variables, such as time of the bed bath and the person providing the bed bath, which are held constant in our study [25].

As part of the laboratory setting, students will be recruited for several reasons. First of all, it would be burdensome for actual bedridden persons to participate in this study, especially given the laboratory setting. Second, the use of students offers advantages not only to the research team (e.g., expected relative ease of access to potential participants) but also enables students to obtain personal experience in bathing methods. Hence, participating students will obtain valuable knowledge for their future careers, which in turn can have positive spillover effects for the patients who will be bathed by these students in the future.

Page 6/10 
We are convinced that the strengths of the laboratory setting with respect to internal validity outweigh its often stated weakness of artificiality [27]. One can argue that the use of students adds to the artificiality of our study because actual patients are generally more vulnerable and nurses spend more time bathing compared to students. However, especially because of these differences between students and actual patients and nurses, it is likely that the differences found between washing without water and the traditional bed bath on comfort among students will also be found among actual patients and nurses. That is, the impact of the bed bath is probably higher for actual patients and nurses compared to students. Nevertheless, although students differ in many ways from actual patients, they are likely to encounter similar feelings of dependency and tension during the bed bath.

\section{Trial Status}

The version number of this protocol is 2.0 (September 13, 2019). Data recruitment started in March 2018 and is expected to be completed by the beginning of 2020 .

\section{List Of Abbreviations}

SPIRIT: Standard Protocol Items: Recommendations for Interventional Trials; CONSORT: Consolidated Standards of Reporting Trials; w-w: washing without water; w+w: traditional bed bath with water and soap; B: Baseline; O: Outcome; PEECE: Patient Evaluation of Emotional Comfort Experienced.

\section{Declarations}

Ethics approval and consent to participate

Ethical approval has been obtained on 16 January 2018 by the Medical Committee METC-Z (17-N-172), which also received the informed consent letter used for this study to ask participants for their consent. Informed consent will be obtained from all study participants. Furthermore, the trial is registered at www.trialregister.nl under the identification number TC6972.

Consent for publication

Not applicable

Availability of data and materials

Materials, such as the bathing instructions used for this study, are available from the corresponding author on reasonable request.

Competing interests

The first author (FMVG) is a doctoral candidate at Maastricht University who is working on research, which is partially funded by Arion Holding B.V. However, employees of this firm were not involved in the collection, analysis, or interpretation of the data. Hence, the authors declare that they have no competing interests.

Funding

This study is partially funded by Arion Holding B.V. (Rutherford 11, 6422 RE Heerlen (NL); info@arion-group.com). However, employees of this firm will not be involved in the collection, analysis, or interpretation of the data, or the decision to submit results.

\section{Authors contributions}

FMVG wrote the protocol and will be responsible for the execution of the study, including recruitment and allocation of study participants, data collection, and data analysis. SMGZ, GO, and JPHH contributed to the development of the study design and study protocol and will provide support in the interpretation of the study results. FT offered statistical support in the power calculation of the sample size and in the selection and execution of appropriate statistical methods in the data analyses.

All authors read and approved the final manuscript.

\section{Acknowledgements}

We would like to thank the coordinators of the participating educational institutions for their support in setting up the research and the recruitment of participants, the research assistants involved in the data collection, and all study participants.

\section{References}

1. Rader J, Barrick AL, Hoeffer B, Sloane PD, McKenzie D, Talerico KA, et al. The bathing of older adults with dementia: easing the unnecessarily unpleasant aspects of assisted bathing. Am J Nurs. 2006;106:40-9.

Page $7 / 10$ 
2. Feo R, Kitson A. Promoting patient-centred fundamental care in acute healthcare systems. Int J Nurs Stud. 2016;57:1-11. doi:10.1016/j.ijnurstu.2016.01.006.

3. Downey L, Lloyd H. Bed bathing patients in hospital. Nurs Stand. 2008;22(34):35-40. doi:10.7748/ns2008.04.22.34.35.c6531.

4. Coyer FM, O'Sullivan J, Cadman N. The provision of patient personal hygiene in the intensive care unit: a descriptive exploratory study of bedbathing practice. Aust Crit Care. 2011;24:198-209. doi:DOI 10.1016/j.aucc.2010.08.001.

5. Lopes JL, Nogueira-Martins LA, de Barros AL. Bed and shower baths: comparing the perceptions of patients with acute myocardial infarction. J Clin Nurs. 2012;22:733-40. doi:10.1111/j.1365-2702.2012.04320.x.

6. Schoonhoven L, van Gaal BG, Teerenstra S, Adang E, van der Vleuten C, van Achterberg T. Cost-consequence analysis of "washing without water" for nursing home residents: a cluster randomized trial. Int J Nurs Stud. 2015;52:112-20. doi:10.1016/j.ijnurstu.2014.08.001.

7. Groven FM, Zwakhalen SM, Odekerken-Schröder G, Joosten EJ, Hamers JP. How does washing without water perform compared to the traditional bed bath: a systematic review. BMC Geriatr. 2017;17:31. doi:10.1186/s12877-017-0425-4.

8. Tutton E, Seers K. Comfort on a ward for older people. J Adv Nurs. 2003;46:380-9.

9. Kolcaba KY. A theory of holistic comfort for nursing. J Adv Nurs. 1994;19:1178-84.

10. Williams AM, Irurita VF. Therapeutic and non-therapeutic interpersonal interactions: the patient's perspective. J Clin Nurs. 2004;13:806-15.

11. Larson EL, Ciliberti T, Chantler C, Abraham J, Lazaro EM, Venturanza M, et al. Comparison of traditional and disposable bed baths in critically ill patients. Am J Crit Care. 2004;13:235-41.

12. Knibbe H, Geuze L, Knibbe N. Ergonomische aspecten van wassen-zonder-water voor zorgverleners. 2005. http://docs.minszw.nl/pdf/92/2005/92_2005_1_9249.pdf. Accessed 13 Aug 2015.

13. Knibbe JJ, Knibbe NE. Businesscase verzorgend wassen wassen-zonder-water. Stichting RegioPlus/Platform Zorginnovatie, Zoetermeer. 2006. http://www.locomotion.nu/doc/innovatiespiegel/BusinesscaseVW.pdf. Accessed 13 Aug 2015.

14. Feo R, Conroy T, Jangland E, Muntlin Athlin $\AA$, Brovall M, Parr J, et al. Towards a standardised definition for fundamental care: a modified Delphi study. J Clin Nurs. 2017; doi:10.1111/jocn.14247.

15. Chan A, Tetzlaff JM, Gøtzsche PC, Altman DG, Mann H, Berlin JA, et al. SPIRIT 2013 explanation and elaboration: guidance for protocols of clinical trials. BMJ. 2013;346:e7586. doi:10.1136/bmj.e7586.

16. Moher D, Hopewell S, Schulz KF, Montori V, Gøtzsche PC, Devereaux PJ, et al. CONSORT 2010 explanation and elaboration: updated guidelines for reporting parallel group randomised trials. BMJ. 2010;340:c869). doi:10.1136/bmj.c869.

17. Protocol "wassen van een zorgvrager op bed". [http://www.factor-e.nl/helpende/images/stories/Helpende/Beroepstaak_7_Wassen_en_aan_en_uitkleden/Protocollen/Protocollen\%20versie\%202/BT\%207\%20Protocol\%20Wassen\%20van\%20een\%20zorgvrager\%20op\%20bed\%20v2.pdf]. Accessed 1 Feb 2018.

18. Hughes R, Huby M. The application of vignettes in social and nursing research. J Adv Nurs. 2002;37:382-86.

19. Williams AM, Lester L, Bulsara C, Petterson A, Bennett K, Allen E, et al. Patient Evaluation of Emotional Comfort Experienced (PEECE): developing and testing a measurement instrument. BMJ Open. 2017;7:e012999. doi:10.1136/bmjopen-2016-012999.

20. de Jonge J, Mulder MJ, Nijhuis FJ. The incorporation of different demand concepts in the job demand-control model: effects on health care professionals. Soc Sci Med. 1999;48:1149-60.

21. Verbeek H. Redesigning dementia care: an evaluation of small-scale, homelike care environments. Maastricht: Maastricht University; 2011.

22. Lachin JM. Introduction to sample size determination and power analysis for clinical trials. Control Clin Trials. 1981;2:93-113.

23. Snijders TA, Bosker RJ. Multilevel analysis: an introduction to basic and advanced multilevel modeling. London: Sage; 1999.

24. Hox JJ. Multilevel analysis: techniques and applications, 2nd ed. New York: Routledge; 2010.

25. Wilson TD, Aronson E, Carlsmith K. The art of laboratory experimentation. In: Fiske ST, Gilbert DT, Lindzey G, editors. Handbook of social psychology. Hoboken: Wiley; 2010. p. 51-81.

26. Mills EJ, Chan AW, Wu P, Vail A, Guyatt GH, Altman DG. Design, analysis, and presentation of crossover trials. Trials. 2009;10:27. doi:10.1186/1745-6215-10-27.

27. Richards DA, Hamers JP. RCTs in complex nursing interventions and laboratory experimental studies. Int J Nurs Stud. 2009;46:588-92. doi:10.1016/j.jinurstu.2008.12.002.

\section{Table}

Table 1 - Outcome overview based on SPIRIT guidelines [15] 


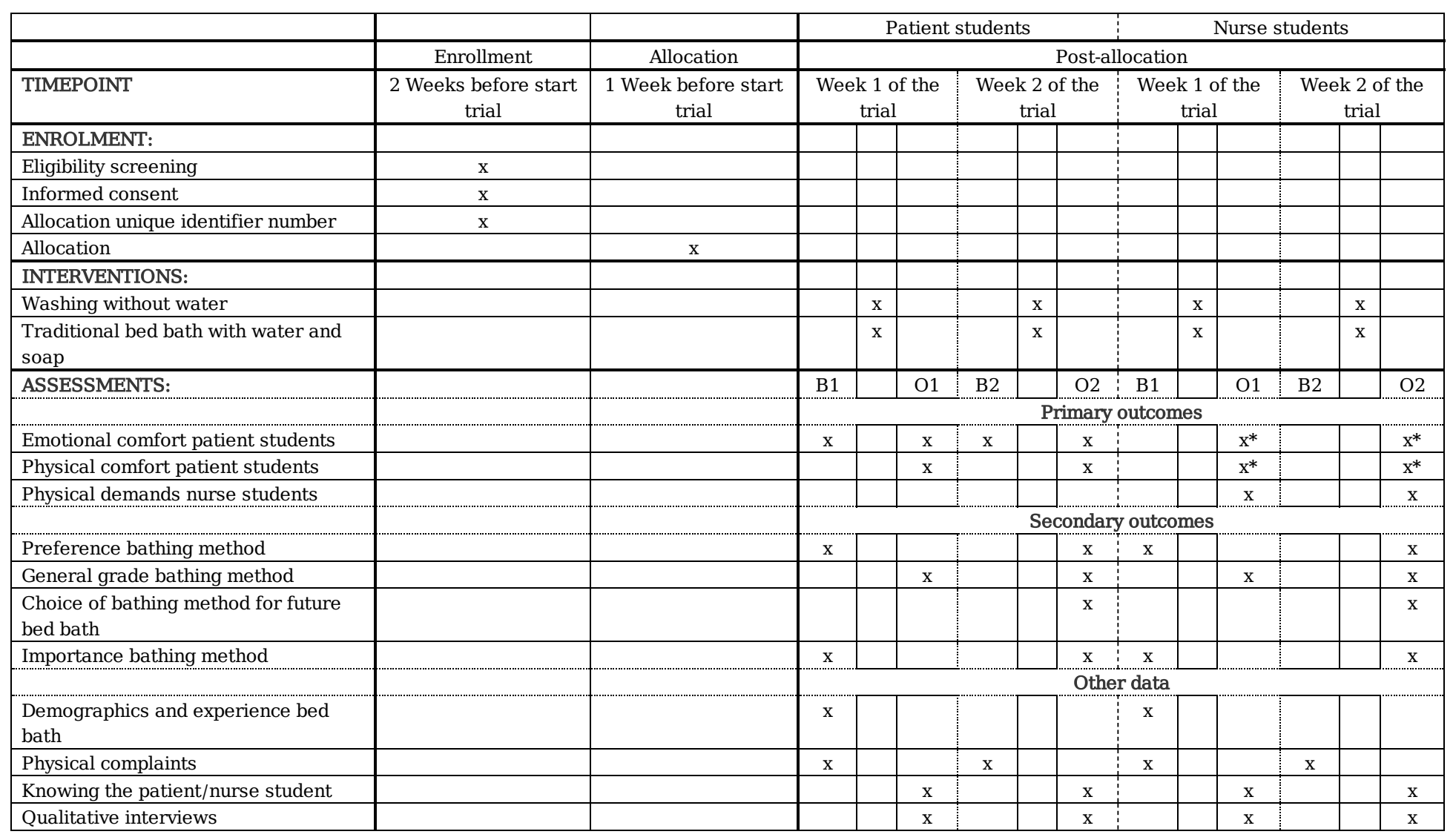

* Emotional and physical comfort for the patient student as perceived by the nurse student are included as secondary outcomes.

Figures 


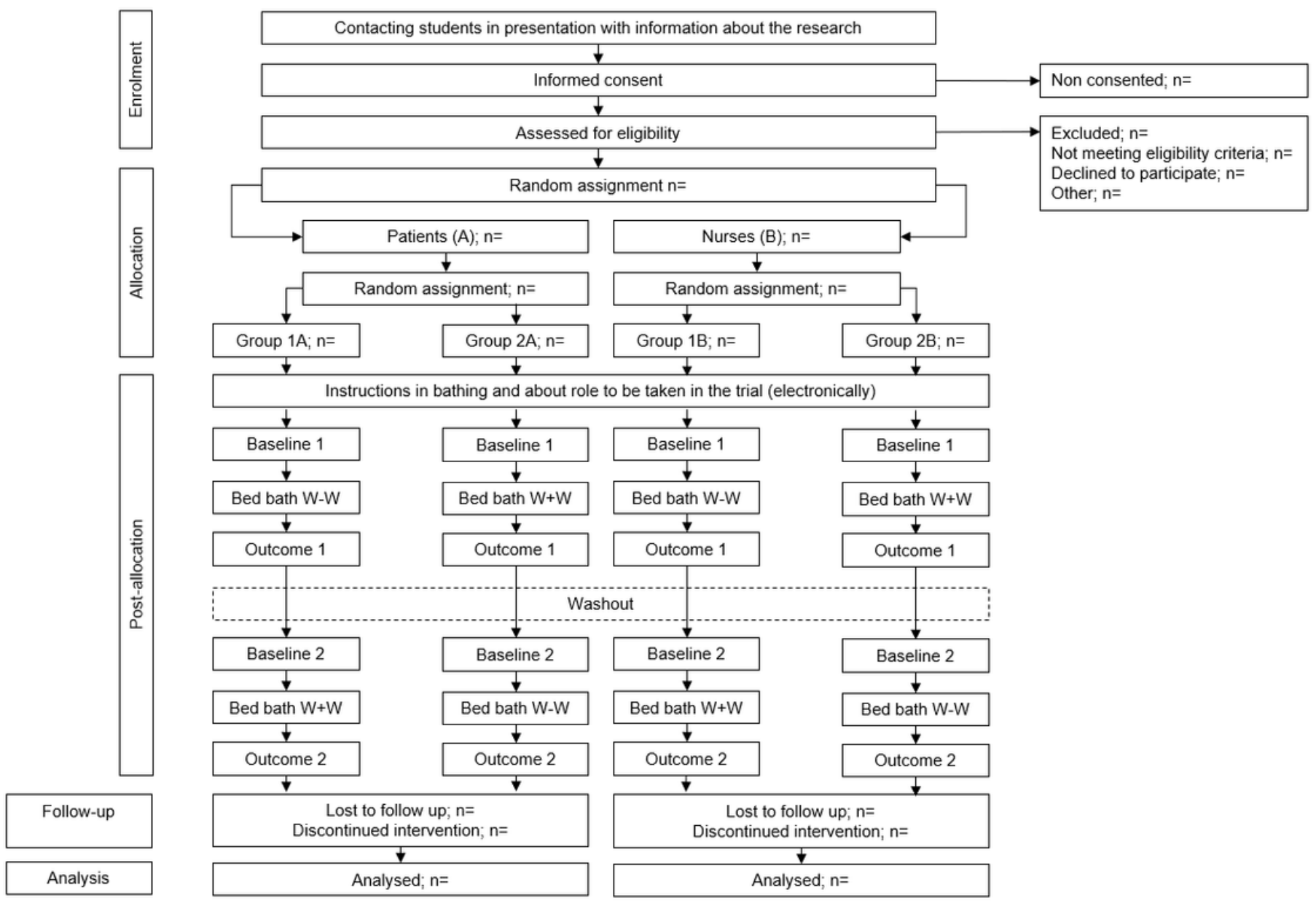

Figure 1

" $\mathrm{W}-\mathrm{W}$ " is a washing without water bed bath and " $\mathrm{w}+\mathrm{W}$ " stands for the traditional bed bath with water and soap. Groups $1 \mathrm{~A}$ and $2 \mathrm{~A}$ (patient students) and $1 \mathrm{~B}$ and $2 \mathrm{~B}$ (nurse students) each differ with respect to the order in which the two bed bathing methods take place. In groups $1 \mathrm{~A}$ and $1 \mathrm{~B}$, students start with the washing without water bed bath, whereas groups $2 \mathrm{~A}$ and $2 \mathrm{~B}$ start with the bed bath with water and soap.

\section{Supplementary Files}

This is a list of supplementary files associated with this preprint. Click to download.

- SPIRITchecklistDSWW.pdf 